\title{
On a generalization of Valiron's inequality for k-hypermonogenic functions on upper half-space
}

\author{
D. Constales*, R. De Almeida ${ }^{\dagger}$ and R. S. Kraußhar** \\ * Department of Mathematical Analysis, Ghent University, Building S-22, Galglaan 2, B-9000 Ghent, Belgium. \\ ${ }^{\dagger}$ Departamento de Matemática, Universidade de Trás-os-Montes e Alto Douro, P-5000-911 Vila Real, Portugal. \\ ** Institut für Mathematik, Universität Paderborn, Warburgerstraße 100, D-33098 Paderborn, Germany
}

\begin{abstract}
We present some results on the asymptotic growth behavior of periodic k-hypermonogenic functions on upper halfspace. A generalization of the classical Valiron inequality for this class of functions and some basic properties are discussed.
\end{abstract}

Keywords: Fourier transform, growth orders, k-hypermonogenic functions, special functions

PACS: 02.30.-f, 02.30.Jr

\section{INTRODUCTION}

For details on Clifford algebras and its function theory we refer for example to [2,9]. Let $\left\{e_{1}, e_{2}, \ldots, e_{n}\right\}$ be the standard basis of $\mathbb{R}^{n}$ and $C l_{n}$ be the associated real Clifford algebra obtained from the generating relations $e_{i} e_{j}+e_{j} e_{i}=-2 \delta_{i j}$, $i, j=1, \cdots, n$ where $\delta_{i j}$ is the Kronecker symbol. Each $a \in C l_{n}$ has the form $a=\sum_{A} a_{A} e_{A}$ with $a_{A} \in \mathbb{R}, A \subseteq\{1, \cdots, n\}$, $e_{A}=e_{l_{1}} e_{l_{2}} \cdots e_{l_{r}}$, where $1 \leq l_{1}<\cdots<l_{r} \leq n$, and $e_{\emptyset}=: e_{0}=: 1$. The Clifford main involution is defined by $e_{0}^{\prime}=1$ and $e_{i}^{\prime}=-e_{i}$ for $i=1, \cdots, n$. The involution is defined by $\widehat{e}_{n}=-e_{n}$ and $\widehat{e}_{i}=e_{i}$ for $i=0, \cdots, n-1$. For any arbitrary $a, b \in C l_{n}$ and $c \in C l_{n-1}$ one has $(a b)^{\prime}=a^{\prime} b^{\prime}, \quad \widehat{a b}=\widehat{a b}, \quad a^{\prime} e_{n}=e_{n} \widehat{a}, \quad e_{n} a^{\prime}=e_{n} \widehat{a}, \quad c^{\prime} e_{n}=e_{n} c, \quad e_{n} c^{\prime}=c e_{n}$. The Clifford conjugate of $a$ is defined by $\bar{a}=\sum_{A} a_{A} \bar{e}_{A}$, where $\overline{e_{A}}=\bar{e}_{l_{r}} \bar{e}_{l_{r-1}} \cdots \bar{e}_{l_{1}}$ and $\bar{e}_{j}=-\bar{e}_{j}, j=1, \ldots, n, \bar{e}_{0}=e_{0}=1$. Any element $a \in C l_{n}$ may be uniquely decomposed as $a=c+d e_{n}$ for $c, d \in C l_{n-1}$. On the basis of this definition one defines the mapping $P: C l_{n} \rightarrow C l_{n-1}$ and $Q: C l_{n} \rightarrow C l_{n-1}$ by $P(a)=b$ and $Q(a)=d$.

The linear subspace $\operatorname{span}_{\mathbb{R}}\left\{1, e_{1}, \cdots, e_{n}\right\}=\mathbb{R} \oplus \mathbb{R}^{n} \subset C l_{n}$ is the so-called space of paravectors $v=x_{0}+x_{1} e_{1}+x_{2} e_{2}+$ $\cdots+x_{n} e_{n}$ which we simply identify with $\mathbb{R}^{n+1}$. The element $x_{0}=: S c(v)$ is called the scalar part of the paravector $v$ while the expression $z:=x_{1} e_{1}+\cdots+x_{n} e_{n}=: \operatorname{Vec}(v)$ is called its vector part. We further indicate its $*$-invariant part by $\underline{z}:=x_{1} e_{1}+\cdots+x_{n-1} e_{n-1}$.

The aim of this paper is to study some questions related to the growth behavior of Clifford valued functions $f(z)=\sum_{A} e_{A} f_{A}(z)$, that satisfy the equation

$$
\left(\sum_{i=1}^{n} \frac{\partial}{\partial x_{i}} e_{i}\right) f(z)+\frac{k}{x_{n}} Q^{\prime} f(z)=\beta f(z), \quad k \in \mathbb{R}, \beta \in \mathbb{R}_{0}^{+}
$$

on upper half-space $H^{+}\left(\mathbb{R}^{n}\right)=\left\{z=e_{1} x_{1}+\cdots+e_{n} x_{n} \in \mathbb{R}^{n} \mid x_{n}>0\right\}$ for general $k \in \mathbb{R}$ and general $\beta \in \mathbb{R}_{0}^{+}$.

Notice if $\beta=0$ the solutions to this equation are called k-hypermonogenic functions and are studied by a constantly growing community, see for instance [1,11, 12, 14]. Furthermore, for $\beta=0$ and $k=0$ the expression (1) is the generalized Cauchy-Riemann equation where the solutions are called monogenic functions and in the case $\beta=0$ where $k=n-1$ the expression (1) is the Dirac-Hodge equation and the solutions are called hypermonogenic functions. The Dirac-Hodge operator is a linearization of the Laplace-Beltrami operator on the upper half-space. This paper provides an extension to [6] where the particular case $k=n-1, \beta=0$ has been treated.

In what follows, we use $\mathbf{m}=\left(m_{1}, \ldots, m_{n-1}\right) \in \mathbb{N}_{0}^{n-1}$ to be an $(n-1)$-dimensional multi-index and $|\mathbf{m}|:=m_{1}+$ $\cdots+m_{n-1}$. 


\section{FOURIER INTEGRAL REPRESENTATION}

The study of the Taylor coefficients of an entire monogenic solution was the basis for the asymptotic growth analysis of entire solutions to partial differential equations (PDEs) related to the generalized Euclidean Dirac and CauchyRiemann operator, see for example [3, 4, 5, 8]. In the context of hypermonogenic functions defined on the upper half-space the study of the Taylor coefficients is replaced by studying their Fourier images, see [6].

Note that one can represent any upper half-space solution to (1) that additionally satisfies

$$
\int_{\mathbb{R}^{n-1}}\|f(z)\| d x_{1} \cdots d x_{n-1}<\infty
$$

by the following Fourier integral

$$
f(z)=\int_{\mathbb{R}^{n-1}} \alpha\left(\underline{w}, x_{n}\right) e^{i\langle\underline{w}, \underline{x}\rangle} d w_{1} \cdots d w_{n-1},
$$

where $\underline{x}=x_{1} e_{1}+\cdots+x_{n-1} e_{n-1}$ and $\underline{w}=w_{1} e_{1}+\cdots+w_{n-1} e_{n-1}$.

In what follows $K_{s}$, for $s \in \mathbb{R}$, denote the usual modified Bessel functions. For its definition and properties, see for instance [13].

Theorem 1 Let $f: H^{+}\left(\mathbb{R}^{n}\right) \rightarrow C l_{n}$ be a solution to (1) satisfying (2). Then for $\underline{w} \neq 0$ the Fourier images of the Fourier integral representation (3) have the following form

$$
\alpha\left(\underline{w}, x_{n}\right)=\phi(\underline{w}) \tilde{\alpha}\left(\beta+\|\underline{w}\|, x_{n}\right)
$$

where precisely

$$
\tilde{\alpha}\left(y, x_{n}\right)=\left(y x_{n}\right)^{\frac{k+1}{2}}\left(K_{\frac{k+1}{2}}\left(y x_{n}\right)+e_{n} K_{\frac{k-1}{2}}\left(y x_{n}\right)\right)
$$

and $\phi(\underline{w})$ is a $C l_{n-1}$ valued function.

Proof. Using the same arguments given in [7], we consider the equation

$$
\left(\sum_{i=1}^{n} \frac{\partial}{\partial x_{i}} e_{i}\right) f(z)+\frac{k}{x_{n}} Q^{\prime} f(z)=\beta f(z), \quad \beta \in \mathbb{R}_{0}^{+} .
$$

Applying the Fourier transform in the first $(n-1)$ components leads to the following differential equation on the Fourier image $\alpha=\alpha\left(\underline{w}, x_{n}\right), \quad \underline{w} \in \mathbb{R}^{n-1}, x_{n}>0$ :

$$
(\underline{i w}-\beta) \alpha+e_{n} \frac{\partial \alpha}{\partial x_{n}}+\frac{k}{x_{n}} Q^{\prime} \alpha=0 .
$$

Splitting $\alpha$ into its $P$-part and $Q$-part $\alpha=P \alpha+e_{n} Q^{\prime} \alpha$ one has

$$
(i \underline{w}-\beta)\left(P \alpha+e_{n} Q^{\prime} \alpha\right)+e_{n} \frac{\partial}{\partial x_{n}}\left(P \alpha+e_{n} Q^{\prime} \alpha\right)+\frac{k}{x_{n}} Q^{\prime}\left(P \alpha+e_{n} Q^{\prime} \alpha\right)=0 .
$$

Since $\alpha$ is a solution to (1), collecting in (6) all terms that include or do not include the factor $e_{n}$, one obtains

$$
(\underline{i} \underline{w}-\beta) P \alpha-\frac{\partial}{\partial x_{n}} Q^{\prime} \alpha+\frac{k}{x_{n}} Q^{\prime} \alpha=0, \quad e_{n}(-i \underline{w}-\beta) Q^{\prime} \alpha+e_{n} \frac{\partial}{\partial x_{n}} P \alpha=0 .
$$

Decomposing $\alpha=A \alpha+\underline{w} B \alpha$ where $A$ and $B$ are appropriately Clifford operators, projecting $\alpha$ to the part of the Clifford algebra which intersects the subspace generated by $\underline{w}$ at 0 , follows

$$
P \alpha=A(P \alpha)+\underline{w} B(P \alpha), \quad Q^{\prime} \alpha=A\left(Q^{\prime} \alpha\right)+\underline{w} B\left(Q^{\prime} \alpha\right) .
$$

Using this decomposition in $P \alpha$ and $Q^{\prime} \alpha$ one rewrites the system (7) in the following way

$$
\frac{\partial}{\partial x_{n}} Q^{\prime} \alpha=\frac{\partial}{\partial x_{n}} A\left(Q^{\prime} \alpha\right)+\underline{w} \frac{\partial}{\partial x_{n}} B\left(Q^{\prime} \alpha\right)=(\underline{i} \underline{w}-\beta) A(P \alpha)-\left(i\|\underline{w}\|^{2}+\beta \underline{w}\right) B(P \alpha)+\frac{k}{x_{n}} A\left(Q^{\prime} \alpha\right)+\frac{k}{x_{n}} \underline{w} B\left(Q^{\prime} \alpha\right)
$$


consequently,

$$
\frac{\partial}{\partial x_{n}} A\left(Q^{\prime} \alpha\right)=-\beta A(P \alpha)-i\|\underline{w}\|^{2} B(P \alpha)+\frac{k}{x_{n}} A\left(Q^{\prime} \alpha\right), \quad \frac{\partial}{\partial x_{n}} B\left(Q^{\prime} \alpha\right)=i A(P \alpha)-\beta B(P \alpha)+\frac{k}{x_{n}} B\left(Q^{\prime} \alpha\right) .
$$

Analogously, one has

$$
\frac{\partial}{\partial x_{n}} P \alpha=\frac{\partial}{\partial x_{n}} A(P \alpha)+\underline{w} \frac{\partial}{\partial x_{n}} B(P \alpha)=-\beta A\left(Q^{\prime} \alpha\right)-i\|\underline{w}\|^{2} B\left(Q^{\prime} \alpha\right)+i \underline{w} A\left(Q^{\prime} \alpha\right)-\beta \underline{w} B\left(Q^{\prime} \alpha\right)
$$

obtaining

$$
\frac{\partial}{\partial x_{n}} A(P \alpha)=-\beta A\left(Q^{\prime} \alpha\right)-i\|\underline{w}\|^{2} B\left(Q^{\prime} \alpha\right), \quad \frac{\partial}{\partial x_{n}} B(P \alpha)=i A\left(Q^{\prime} \alpha\right)-\beta B\left(Q^{\prime} \alpha\right) .
$$

In the case $\underline{w} \neq 0$ the general solution of the system (8) and (9) is

$$
\left.\alpha\left(\underline{w}, x_{n}\right)=x_{n}^{\frac{k+1}{2}} C_{4}^{*}(\underline{w})\left(K_{\frac{k+1}{2}}\left((\beta+\|\underline{w}\|) x_{n}\right)+e_{n} K_{\frac{k-1}{2}}\left((\beta+\|\underline{w}\|) x_{n}\right)\right)\right)
$$

Notice that this representation also remains valid for $w=0$ due to the proper asymptotic behavior of the Bessel $\mathrm{K}$ functions at zero.

In particular, one obtains that every solution of the equation (1) on the upper half-space that is $(n-1)$-fold periodic with respect to the orthonormal standard lattice $\Lambda^{n-1}=\mathbb{Z} e_{1}+\cdots+\mathbb{Z} e_{n-1}$ has a discrete Fourier series representation of the form

$$
f(z)=\frac{1}{(2 \pi)^{n}} \sum_{\underline{w} \in \Lambda^{n}} \alpha\left(2 \pi \underline{w}, x_{n}\right) e^{-2 \pi i\langle\underline{w}, \underline{x}\rangle} .
$$

where $\quad \alpha\left(2 \pi \underline{w}, x_{n}\right)=\left((\beta+2 \pi\|\underline{w}\|) x_{n}\right)^{\frac{k+1}{2}} \phi(2 \pi \underline{w})\left(K_{\frac{k+1}{2}}\left((\beta+2 \pi\|\underline{w}\|) x_{n}\right)+e_{n} K_{\frac{k-1}{2}}\left((\beta+2 \pi\|\underline{w}\|) x_{n}\right)\right)$.

This representation is valid for all functions that are solution to (1) on the upper half-space.

\section{Growth orders and analogues of the maximum term and the central index}

The aim is to develop analogues to some basic results from Wiman and Valiron's $[15,16]$ theory for solutions of (1) on the upper half-space. Under this point of view we introduce

Definition 1 Let $f: H^{+}\left(\mathbb{R}^{n}\right) \rightarrow C l_{n}$ be a solution of (1) with Fourier integral representation as described in Theorem 1 . Then its order of growth and lower order of growth are defined respectively by

$$
\rho(f):=\limsup _{\|\underline{w}\| \rightarrow+\infty}-\frac{\|\underline{w}\| \log \|\underline{w}\|}{\log \|\phi(\underline{w})\|}, \quad \lambda(f):=\liminf _{\|\underline{w}\| \rightarrow+\infty}-\frac{\|\underline{w}\| \log \|\underline{w}\|}{\log \|\phi(\underline{w})\|} .
$$

If $\rho(f)=\lambda(f)$, then $f$ is a function of regular growth. If $\rho(f)>\lambda(f)$, then $f$ has irregular growth.

Remark: Due to this definition we may construct for arbitrary order of growth, examples of $(n-1)$-fold periodic functions which satisfies (1) using its discrete Fourier series representation on upper half-space. by

We define for $(n-1)$-fold periodic solution of (1) with a discrete Fourier series of the form (10), the maximum term

$$
\mu\left(x_{n}, f\right):=\max _{\underline{w} \in \Lambda^{n-1}}\left\{\left\|\alpha\left(2 \pi \underline{w}, x_{n}\right)\right\|\right\} .
$$

The notion of the central index $v\left(x_{n}, f\right)$ is equal to the value of $|\underline{w}|$ where $\underline{w}$ is (are) exactly that (those) discrete lattice point(s) for which the equality $\left\|\alpha\left(2 \pi \underline{w}, x_{n}\right)\right\|=\mu\left(x_{n}, f\right)$ is attained.

In this context, we are much closer to the theory of entire monogenic functions, in which one considers discrete Taylor series. The lattice points $\underline{w}$ then correspond to the multi-indices $\mathbf{m} \in \mathbb{N}_{0}^{n-1}$ over which the summation of the multiple Taylor series is extended.

Proposition 1 Suppose that $f: H^{+}\left(\mathbb{R}^{n}\right) \rightarrow C l_{n}$ is a solution of (1) and (n-1)-fold periodic with respect to the orthonormal lattice $\Lambda^{n-1}$. Then there exists an $X_{n}>0$ such that $\mu\left(x_{n}, f\right)$ decreases for $x_{n} \leq X_{n}$ strictly monotonic. 
In this result, analogous to the one given in [6], one can see that $\mu\left(x_{n}, f\right)$ is strictly monotonic decreasing. This is an important qualitative difference to the case of dealing with entire monogenic function whose maximum term $\mu(r, f)$ tends to infinity if $r$ tens to infinity.

Using Proposition 1, one proves an upper bound estimate of the maximum modulus which we regard as a generalization of the classical Valiron inequality for holomorphic entire functions.

Theorem 2 Suppose that $f$ is an $(n-1)$-fold periodic solution of $(1)$ on $H^{+}\left(\mathbb{R}^{n}\right)$ with period lattice $\Lambda^{n-1}$. Then there exists real constants $L$ and $X_{n}$ such that for all $0<y_{n}<x_{n} \leq X_{n}$ :

$$
M\left(x_{n}, f\right) \leq \mu\left(x_{n}, f\right)\left(\left(2 v\left(x_{n}, f\right)-1\right)^{n}+2^{n} L\left(\frac{x_{n}}{y_{n}}\right)^{\frac{k-1}{2}} e^{-\frac{2 \pi}{\sqrt{n}} v\left(y_{n}, f\right)\left(y_{n}-x_{n}\right)}\left(1-e^{-\frac{2 \pi}{\sqrt{n}}\left(x_{n}-y_{n}\right)}\right)^{-n}\right) .
$$

Notice that this inequality does not depend on the particular choice of $\beta$.

\section{ACKNOWLEDGMENTS}

The first author's research is supported by BOF/GOA 01GA0405 of Ghent University. The second author was partial supported by the R\&D unit Centro de Investigação e Desenvolvimento em Matemática e Aplicações (CIDMA) of the University of Aveiro, through the Portuguese Foundation for Science and Technology (FCT), co-financed by the European Community fund FEDER.

\section{REFERENCES}

1. S. Bernstein, S.-L. Eriksson, Qiao Yuying and J. Ryan. Function theory for Laplace and Dirac-Hodge operators on hyperbolic space. Journal D'Analyse Mathématique 98 2006, 43-64.

2. $\quad$ F. Brackx, R. Delanghe and F. Sommen. Clifford Analysis. Pitman 76, London, 1982.

3. D. Constales, R. De Almeida and R.S. Kraußhar. On Cauchy estimates and growth orders of entire solutions to iterated Dirac and generalized Cauchy-Riemann equations. Mathematical Methods in the Applied Sciences 29 No. 14, (2006), 1663-1686.

4. D. Constales, R. De Almeida and R.S. Kraußhar. Further results on the asymptotic growth of entire solutions of iterated Dirac equations in $\mathbb{R}^{n}$. Mathematical Methods in the Applied Sciences 29 No. 5 (2006), 537-556.

5. D. Constales, R. De Almeida and R.S. Kraußhar. Applications of the maximum term and the central index in the asymptotic growth analysis of entire solutions to higher dimensional polynomial Cauchy-Riemann equations. Complex Variables and Elliptic Equations 53 No. 3 (2008), 195-213.

6. D. Constales, R. De Almeida and R.S. Kraußhar. On the growth behavior of hypermonogenic functions on upper half-space. Simos, Theodore E. (ed.) et al., Numerical analysis and applied mathematics. International conference on numerical analysis and applied mathematics, Rethymno, Crete, Greece, September 18-22, 2009. Vol. 2. Melville, NY: American Institute of Physics (AIP). AIP Conference Proceedings 1168, 2 (2009), 757-760.

7. D. Constales, R.S. Kraußhar and J. Ryan. k-hypermonogenic automorphic forms. Journal of Number Theory, 126 (2007), 254-271.

8. R. De Almeida and R.S. Kraußhar. On the asymptotic growth of monogenic functions. Zeitschrift für Analysis und Ihre Anwendungen 24 No. 4 (2005), 763-785.

9. R. Delanghe, F. Sommen and V. Souček. Clifford Algebra and Spinor Valued Functions. Kluwer, Dordrecht-Boston-London, 1992.

10. S.-L. Eriksson-Bique and H. Leutwiler. Hypermonogenic functions and Möbius transformations. Advances in Applied Clifford Algebras 11 (S2) (2001), 67-76.

11. S.-L. Eriksson. Integral formulas for hypermonogenic functions. Bulletin of the Belgian Mathematical Society - Simon Stevin 11 No.4 (2005), 705-718.

12. S.-L. Eriksson, Cauchy-type integral formulas for k-hypermonogenic functions. Begehr, H. G. W. (ed.) et al., More progresses in analysis. Proceedings of the 5th international ISAAC congress, Catania, Italy, July 25-30, 2005. Hackensack, NJ: World Scientific. 1051-1064 (2009).

13. I. Gradshteyn and I.M. Ryzhik. Table of Integrals Series and Products. New York: Academic Press 1980.

14. H. Leutwiler. Modified Clifford Analysis. Complex Variables 17 (1992), 153-171.

15. G. Valiron. Lectures on the general theory of integral functions. Chelsea, New York, 1949.

16. A. Wiman. Über den Zusammenhang zwischen dem Maximalbetrage einer analytischen Funktion und dem größten Gliede der zugehörigen Taylorschen Reihe. Acta Mathematica 37 (1914), 305-326. 